\title{
Stochastic decision model of consumer product preference on
} Ecommerce

Vinayagam.P \#1 $^{\text {, Dr.Iyem Perumal }}{ }^{\# 2}$. A, Dr.Rajagopalan, S.P ${ }^{\# 3}$, Dr.S.Dheva Rajan ${ }^{\# 4}$

${ }^{1,2}$ Professor, Department of Mathematics

${ }^{3}$ Department of Computer Science

Dr. MGR Educational and Research Institute, University, Chennai, Tamil Nadu, India.

${ }^{4}$ Department of Information Technology, Sultanate of Oman

\section{ABSTRACT}

Through purchasing electronically is a famous preference now a day among people across the borders. Here, it is presented a decision tree model with stochastic parameters to analyze the item preference of a consumer especially in ecommerce market.

Keywords: Ecommerce, Stochastic, consumer, model, decision making.

\section{Corresponding Author: Vinayagam $\mathbf{P}$}

\section{INTRODUCTION}

Among the three countries in Hawk's study, India seems in the least favorable position for the growth of e-commerce. According to Hawk (2004) India was home to only 5 million Internet users, which is about 0.5 percent of the population. India has the largest offshore development industry of any Country with annual revenue of approximately $\$ 10$ billion [5]. Mathematical modelling is a process by which a real world problem is described by a mathematical Formulation [11]. People are using mathematical modeling even for the prediction of diseases, then why not for the use of security. For an instance, recently, Dheva Rajan et al., [6] proposed a model for dengue. The existence was also analyzed again by Dheva Rajan et al in 2014 [7]. The changes in the solution while changing the parameters of the equations was studied for the above said model also discussed again by Dheva Rajan et al in 2014 [8]. Dheva Rajan [9] did the analysis on the sensitivity of the parameters defined. Srinivasan, V et al., 2014 [13] proposed stochastic modeling and again Srinivasan, V et al., 2014 [13] proposed deterministic model for the prediction. The aim of the paper is mathematically analyzing and establish the two main factors of such systems, namely, waiting time and response time, thereby improving the overall throughput of the system. [12]

\section{THE DECISIOIN PROCESS}

By assuming the following parameters, one can create a standard logit model for the customer preference. Let a given item $\mathrm{i}$ has been chosen by a customer to purchase. The different items numbered from 1 to $\mathrm{n}, \mathrm{n}$ should be a positive integer $p_{i}$ is the choice of that customer assumes the likelihood $v_{i}$ is the value of the customer attaches to the item ; $P_{i}$ is the price of the item, $\mathrm{s}$ is the measure of the importance relatively with the price and the customer. Let $\mathrm{C}$ be a normalization constant. Then the standard logit model is given by,

$$
p_{i}=C e^{\left(V_{i}-s P_{i}\right)}
$$

with sum of pi is equal to 1 always that is, where 


$$
\sum_{i=1}^{n} p_{i}=1
$$

$\mathrm{i}$ varies from 1 to $\mathrm{n}$; If the impact on the consumer of the quality of the item is $V_{i} \cdot Q_{i}$, the improved possibility of the consumer purchasing the similar kind of item as he bought earlier. This may happen usually since the consumer is always influenced by his family and friends circle and the earlier experience from the item.

One can consider an indicator function $I_{i}$ as follows.

$$
I_{i}=\left\{\begin{array}{c}
0, \\
\text { if thecustomerbought the } \\
\text { product'i'already } \\
0, \text { Otherwise }
\end{array}\right\}
$$

Consider $N_{i}$ is the number of family and friends who already preferred/bought/experienced the item $i$. Let a be the constant measuring the relative strength of astuteness

Let $l$ be the constant measuring the relative strength loyalty; Let $h$ be the constant measuring the relative strength of herding. Hence, these dependences may be taken as a linear equation as follows.

$$
V_{i}=a Q_{i}+l I_{i}+h N_{i}
$$

The main assumption in this model is, all the items are independent. The one and only link is the likelihood happens by $\mathrm{C}$, the normalization constant. Few can propose that there is an indication that customers do not appraise all merchandises independently in this way, but obviously make the judgements between merchandises when concluding which to purchase. Thus the likelihood of purchasing the item $i$ will hang on not only on the worth $\left(V_{i}\right)$ and charge of that item $\left(P_{i}\right)$, but on the worth and charge of all the goods. The goal of this section is to formulate a model for this likelihood which is based on binary comparisons, that is, on (possibly successive) comparisons of two items at a time.

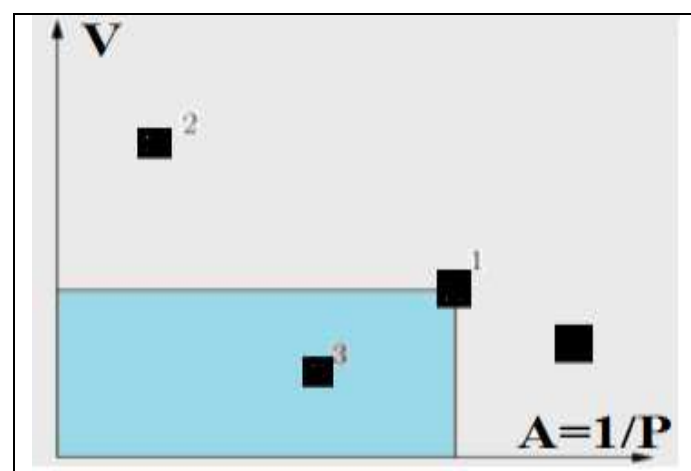

Fig 1: Items are in the affordabilityvalue plane. The colored region is the region of items subjugated by item 1 .

\section{BINARY COMPARISIONS}

Suppose a consumer selects to match item $i$ with item $j$. What may be the likelihood that he/she will choose $i$ over $j$ ? In the Logit Model One would simply have

$$
\begin{gathered}
p_{i}=C e^{\left(V_{i}-s P_{i}\right)} \text { and } \\
p_{j}=C e^{\left(V_{j}-s P j\right)}
\end{gathered}
$$

where $C$ is selected to preserve the equality, $p_{i}+p_{j}=1$. 
An another method would be to assume that the likelihood depends on the difference in value and the difference in price of the two goods. Also assume that, $\mathrm{F}$ is some function which maps to $(-\infty, \infty)$ to $[0,1]$. This can be written as,

$$
p_{i}=1-p_{j} p_{i}=F\left(\left(V_{i}-V_{j}\right)-s\left(P_{i}-P_{j}\right)\right)
$$

for instance, a One well known example is

$$
F(x)=\frac{(1+\tanh x)}{2} .
$$

Dominance is one another idea to consider the position of the items especially in the affordability value plane. One can assume without loss of generality that affordability

$$
A=1 / \text { price }
$$

For an instance, consider the example the three merchandises shown in the following Picture. 1. If one attempts and match items 1 and 2, one can understand that the item 2 has an inferior affordability (i.e. a greater cost) than item 1, but it also has a greater value. Thus the customer has to make a decision on which of value and price is the most important. On the other hand, if one matches items 1 and 3 , item 3 is of lesser value than item 1 , and has a lesser affordability, and thus set a choice among item 3 and item 1, a balanced consumer should choose item 1 at all the time.

For the comparisons among the items where neither is dominated, one can use either the Logit model or the alternative model. To check the quantity of likelihoods that a model gives increase to the likelihoods of selecting each item one requires to build a decision tree.

The decision tree is simple to construct and easy to understand. In creating the decision tree one have to select which two goods the customer will pick to match. It is assumed primarily for easiness that this decision is homogenously unsystematic. Hence, there is an identical likelihood of selecting each couple. Once also have to select the number of comparisons the customer will make. the choice of which items to compare, and the outcome of the comparison yields two stages, in each level of the decision tree.

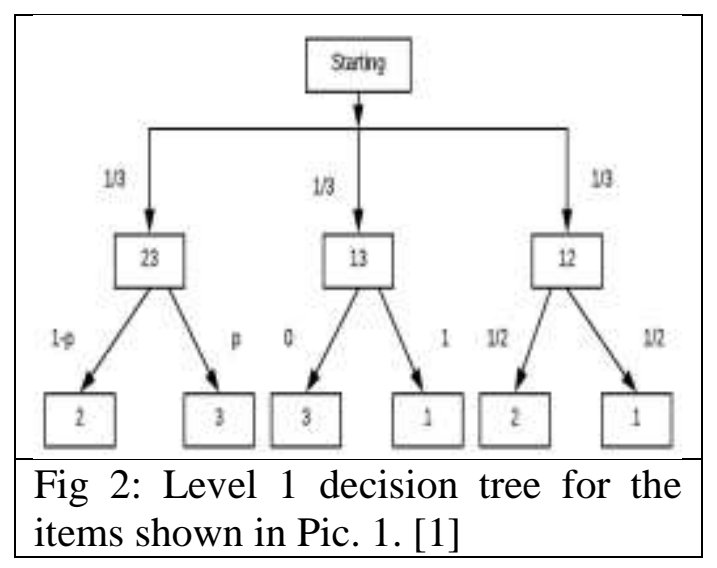

\section{LEVEL 1 DECISION TREE}

The naivest decision tree model for the 3 items are shown in Pic. 1, with the level of comparison one. Level of comparison 1 means that the customer purchases after comparing exactly two items and that is given in Pic. 2. One now can assume that the items 1 and 2 are equally attractive to the customer, hence in the absence of item 3 each would have a likelihood of selection of $1 / 2$. After, one can realize the consequence of the effect that the inclusion of item 3 does to this balance of likelihood. On our pricing page, the user decides whether he/she wishes to purchase our product. This page will shape his/her entire perception of our product. Our pricing page is where the most important action takes place. How can you 
help users choose the right option? More precisely, how can you nudge user toward the option that you want them to choose? Without being too sinister, it is possible through some psychological savvy to compel people to take action on the pricing option that you prefer. It's called the decoy effect. [4] and in particular to the above one, whether there is a decoy effect, that is, since item 1 overlooks item 3, the inclusion of item 3 might mean that more people will select to purchase item 1 . In this situation item 3 acts as a decoy, routing customers to item 1. Let us assume that the likelihood of selecting item 3 over item 2 in a comparison is $p$.

$$
\begin{aligned}
p_{1} & =(1 / 3) *(1 / 2)+(1 / 3) * 1=0.5 \\
p_{2} & =(1 / 3) *(1 / 2)+(1 / 3) *(1-p) \\
& =(1 / 2)-(p / 3) \\
p_{3} & =(1 / 3) *(0)+(1 / 3) * p=(p / 3)
\end{aligned}
$$
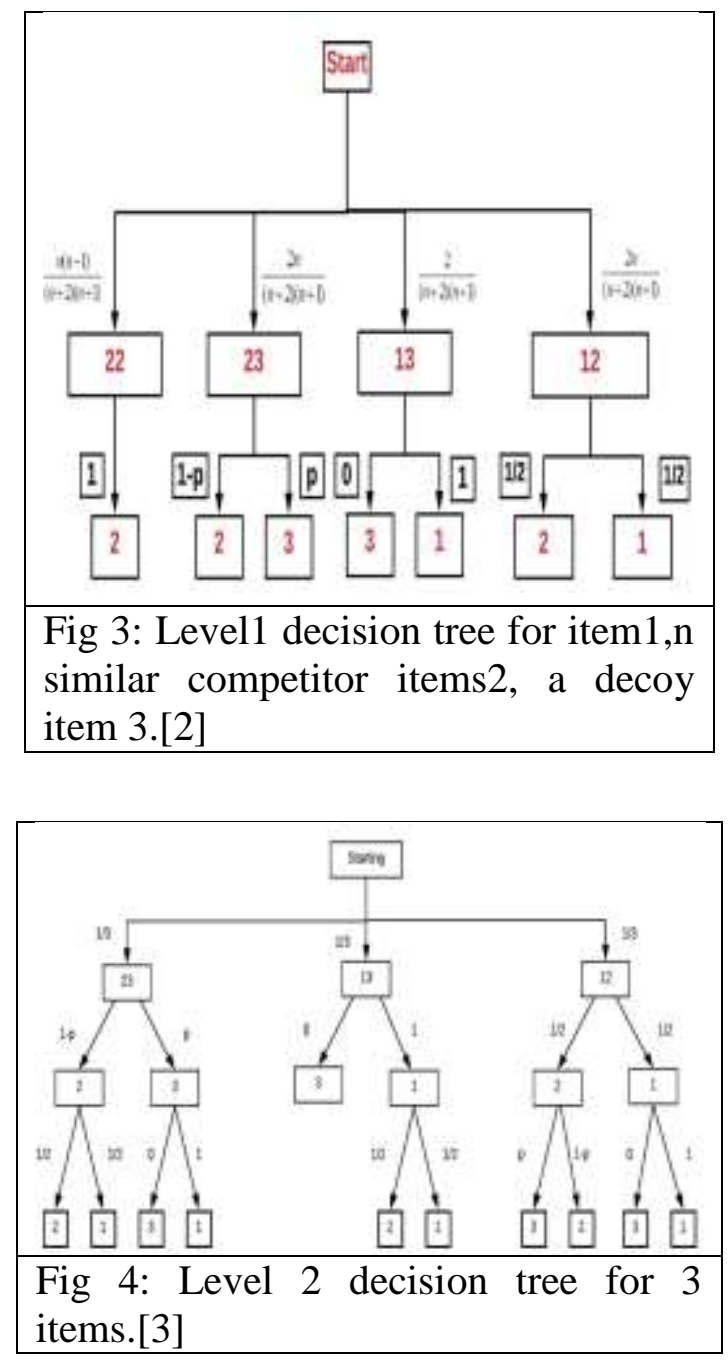

The likelihood of reaching any leaf in the decision tree is simply the item of the likelihoods of taking each division required to develop there. Hence, after introducing item 3 the likelihoods of selecting each item are given above.

Thus, one can understand that in this simple model there is no decoy effect; the likelihood of selecting item 2 has reduced, but the likelihood of selecting item 1 is the identical as it was already existing when item 3 was introduced. Thus there is a chance that the consumer does not even choose to examine item 1, and this exactly offsets the effect of the decoy. 
A simple alteration of this model can be utilized to ruminate the effect of a decoy on item 1 when there is more than one challenger. Supposing that instead of one challenger (item 2) there are $n$ competitors to item 1 , and that all the competitors are equivalent from the point of view of the consumer. Then one can protuberance all the competitors into a single item number 2, with the main change to the decision tree being to the chance of selecting items to compare. Of course it is now possible that the consumer chooses to compare two competitor's items, and one must take this into account. The new decision tree is shown in Pic. 3. The likelihoods of selecting each item are given below. Hence, again, one can understand that there is no decoy effect; item 1 has exactly the same market share as it would have if item 3 is not present.

$$
\begin{aligned}
p_{1}= & \frac{2 n}{(n+2)(n+1)} \times \frac{1}{2}+\frac{2}{(n+2)(n+1)} \times 1 \\
= & \frac{1}{(n+1)} \\
p_{2}= & \frac{2 n}{(n+2)(n+1)} \times \frac{1}{2} \\
& +\frac{2 n}{(n+2)(n+1)} \times(1-p) \\
& +\frac{n(n-1)}{(n+2)(n+1)} \\
= & \frac{n}{(n+1)}-\frac{2 n p}{(n+2)(n+1)} \\
p_{3}= & \frac{2 n p}{(n+2)(n+1)}
\end{aligned}
$$

\section{LEVEL2 DECISION TREE}

One now can get back to 3 items, and adopt that the customer does not stay at one level of comparison, but takes the winner of the first comparison and matches it with the remaining item. In this case One have the level 2 decision tree shown in Pic. 4. Understand that for the second comparison there is no choice of items to match, since there is only one item remaining.

The likelihoods of selecting each item are now

$$
\begin{array}{rlr}
p_{1} & =(1 / 6)+(1 / 6)+(p / 3)=(1-p) / 6) \\
& =(1 / 2)+(p / 6) \\
p_{2} & =(1-p) / 6+(1 / 6)+(1-p) / 6 \quad p_{3}=(p / 6) \\
& =(1 / 2)-(p / 3)
\end{array}
$$

Thus in this case the market shares of item 1 does increase, so that there is a decoy effect.

\section{Conclusion}

Here it is discussed the stochastic parameters of the decision tree to analyze the item preference of a consumer especially in ecommerce market. The choices are given till three and it can be extended to $\mathrm{n}$ numbers. The generalizations may be proved using induction method. As a future work, a similar work can be done for suppliers. The above work can be done with restrictions of consumer preferences. 


\section{REFERENCES}

I. Carmel, E. 1999, 'Global Software Teams: Collaborating Across Borders and Time Zones', Prentice-Hall.

II. Dheva Rajan, S, Iyem perumal, A, Rajagopalan, SP \& Kalpana, D 2013, 'SPR SODE Model for dengue fever', International Journal of Applied Mathematical and Statistical Sciences, vol. 2, no. 3, pp. 41-46.

III. Dheva Rajan, S, Iyem perumal, A, Rajagopalan, SP \& Kalpana, D 2014a, 'Existence of the solution and disease free equilibrium of SPR_SODE Model', International Journal of Pure and Applied Mathematical Sciences, vol. 7, no. 1, pp. 1-7.

IV. Dheva Rajan, S, Iyem perumal, A, Rajagopalan, SP \& Kalpana, D 2014b, 'Bifurcation Analysis of SPR_SODE Model for the spread of dengue', International Journal Applied Engineering Research, vol. 9. no. 6, pp. 643-651.

v. Dheva Rajan, S, Iyem perumal, A, Rajagopalan, SP \& Kalpana, D 2014c, 'Sensitivity Analysis of SPR_SODE Model for the spread of dengue', International Journal of Applied Environmental Sciences, vol.9, no. 4, pp. 1237-1250.

vi. Hawk, S. (2004). A Comparison of B2C E-Commerce in Developing countries. Electronic Commerce Research, 4(3), 181-199.

VII. Murthy, D Page,N \& Rodin, E 1990, 'Mathematical Modelling - A Tool for Problem Solving in Engineering, Physical, Biological and Social Sciences', Pergamon Press, Headington Hill Hall, Oxford, New York.

vIII. Riktesh Srivastava, Dr. Rashad Al-Saed 2012, 'Mathematical model using BI for improving E-Commerce Businesses applicability's', The research journal of Science and IT management, vol. 1, no 10, pp.137-162.

IX. Srinivasan, V Dheva Rajan, S Iyem perumal, A \& Rajagopalan, SP 2014 a, 'An SPR_SM to Predict Dengue Fever', International Journal of Applied Environmental Sciences, vol. 9, no. 4, pp. 2233-2242.

x. https://www.lucidchart.com/documents/edit/e393b6e1-6acd-4aaf-97af-4413d7cb842e\#

XI. https://www.lucidchart.com/documents/edit/62615228-b391-46f5-a0cd-361cefe9cbe2\#

XII. https://www.lucidchart.com/documents/edit/e393b6e1-6acd-4aaf-97af-4413d7cb842e\#

XIII. https://www.jeremysaid.com 